\title{
The Demand Situation Investigation and Development Strategy of the Community Education of the Migrant Workers in Guangzhou
}

\author{
Lu Lu \\ Department of public management, Guangzhou City Polytechnic, Guangzhou, 510408, China
}

\begin{abstract}
Key Words: Migrant workers, Community, Study demand, Problem analysis, Policy suggestion
\end{abstract}
\begin{abstract}
The migrant workers have become the main employment power of city development in Guangzhou. In this research, it chooses the employment area with the concentrated migrant workers to collect data through field questionnaire. By adopting the analytical method of SPSS software statistics, it gains relevant empirical support and puts forward the development strategy and policy suggestion based on the community education demand of the migrant workers in Guangzhou.

In Guangzhou, the migrant workers have taken up half of the population. They create the wealth for the city development and have become the main force which drives the city economy and social development in Guangzhou. It is more beneficial for the incorporation of migrant workers into the team of city builders in Guangzhou by improving the working environment of the migrant workers, strengthening vocational training by taking the community courses as the pointcut and lifting the employability. In this way, they play their role more positively.
\end{abstract}

\section{I.The Demand situation of the Community Education of the Migrant Workers in Guangzhou}

(1). The Status Quo and the Development Trend of the Number of Migrant Population Flow in Guangzhou

At 12 o'clock(the standard time for the census) at midnight on the first day of November in 2010, the permanent population is 12.7008 million which increases by 2.7578 million people compared with the fifth national census as 12 o'clock at midnight on the first day of November. The total growth in ten years is $27.74 \%$ and the annual average growth rate is $2.48 \%$ which is higher than the average level: national(4.67\%), provincial(1.90\%), but lower than the rate of the last ten years, which is 4.67\%(1990-2000). The speed increase of the migrant workers in Guangzhou is higher than that of the registered population. The proportion of migrant workers in permanent population is raised to $37.48 \%$ from $33.29 \%$ in 2000 , which is main cause of the rapid increase of the overall population size in Guangzhou.

(1). Basic Status Analysis of the employment of the Migrant Workers.

Among the respondents, the percentage of men and women is similar with men taking up $54.25 \%$ and women occupying $45.75 \%$. The amount of men is higher than that of the women; the respondents whose ages are above 50 only hold $2.75 \%$, the migrant workers' age all varies from 20 to 50, which takes up $85.5 \%$ of all the respondents. Among the ages from 20 to 50, respondents whose ages vary from 20 to 30 are the primary and they occupy $53.25 \%$ of all the respondents with the unmarried taking up 52\% , the married $45.25 \%$ and the divorced $2.75 \%$. Of all respondents, there is a big proportion of people who are unmarried; People with monthly income below 1000 yuan occupy 5.75\%, between 1000 yuan and 2000 yuan, 22\%, between 2000 yuan and 3000 yuan, $31 \%$, between 3000 yuan and 4000 yuan, 23.35\%, and above the 4000 yuan, 18\%. Among them, a small number of migrant workers approve that some costs should be paid to join the community course training, but for most migrant workers, the cost available for the training and study generally remains low, which has turned into one of the impediment of the study demand of community course; among the respondents, people with the residence within 3 years take up 32.5\%, 3 to 5 years, $31.75 \%, 6$ to 10 years, $21,75 \%$ and over 10 years, $24 \%$.

(2). The Demand Situation Analysis of the Cultural Quality and Spare-time Culture of the Migrant Workers.

The statistics of questionnaire frequency shows that the respondents whose education level is primary school education take up 7.25\%, junior high school, 25.75\%, senior high school or 
technical secondary school, $29.75 \%$, junior college, $19.25 \%$, bachelor degree or above it, $18 \%$ and of all, respondents with cultural level being the junior or senior high school are the main trend. The data analysis shows that the learning period of half population who receive the basic education before they come to Guangzhou is obviously lower than the Guangzhou's requirement toward the migrant workers; the cultural and recreational activities participated by respondents with different gender presents different characters, but more than 50\% of the migrant workers of various ages and gender choose the same options, such as 'physical exercise', 'watching TV', 'surfing the internet' or 'go shopping' in which men prefer 'physical exercise' and 'surfing the internet' more, while women prefer more about 'watching TV' and 'go shopping'.

(3).The Analysis of the migrant workers' Attitude and Demand of Community Education Course and the Understanding of Community Education

The questionnaire investigation of the demand of the community course and training starts its investigation from four points which are, whether their community provides the community course, whether they have participated in the course training, whether they know about the community education course provided by the community and whether it is necessary to launch the community course education. It mainly investigates the basic situation of the launch of the community course in the research area at present and the migrant workers' familiarity of the community course. The questionnaire frequency statistics shows that the migrant workers who choose the generality of the community education training demand take up 50\% and there is equal proportion for the selection: 'necessary to launch', 'it doesn't matter'. It shows that although the migrant workers have demand toward the community education, but their enthusiasm for participation is low.

(4).The Content and Form Analysis of the Migrant Workers' Community Education Course Demand

The questionnaire investigation of the professional skills elevation mainly studies the migrant workers' demand degree toward various skills in their operating post at present to lay a positive foundation for the development of community course. The manifestation of the questionnaire frequency statistic is the following:

The first is the working skill. Most respondents think that the skills need improvement are 'computer skills', 'management skills', 'social skills' and 'the theoretical knowledge related to the industry'. Among the respondents, most men need 'the theoretical knowledge related to the industry', 'computer skills' and 'management skills' while half women need to lift their 'social skills'

The second is the living skill. Most respondents need to improve their 'basic knowledge about the foreign language(social foreign language)', 'Cantonese(local language)', 'mental health knowledge'. Women are more in urgent need of these needs.

\section{The Analysis of the Main Obstacle of the Community Education Training for the Migrant Workers.}

(1) The Problem of Cost-sharing of the Community Education

Who share the education cost is the core problem of the migrant workers' professional skill lifting. The government does not invest enough public finance and the employers are not willing to pay for it due to the cost efficiency. For the migrant workers, although they have strong desire, they do not have the extra money to shoulder it limited by their economic condition. Thus it is imperative to form an efficient educational cost-sharing system with the cost being shared by the government, enterprise and the migrant workers.

(2). The Quality Problem of the Community Education Course

Through investigation, we find that the design of the course content and teaching method are still far from the real demand of the migrant workers due to the lack of first-hand research of the migrant workers in the community courses having been launched in Guangzhou.

In the first place, the existent training contents of community course do not have strong pertinence. At present, the training for migrant workers in Guangzhou mainly focus on the training of junior staff in service and the importance attached to the technical vocational training on demand 
needed in the growing logistics, internet, automobile, construction and municipal administration is not enough.

In the second place, the teaching effect is not obvious. The phenomena such as single teaching form, lack of training equipment and the hardware for the site, low-level faculty and one does not do one has learned, what he has learned has nothing to do with the job he takes, are very serious.

In the third place, the organization method for teaching is single and there exists short-term behavior. The short-term induction training provided by the 'employment units' takes the dominant position while the vocational colleges connected closely with the occupation post rarely join the training of professional skill guided by the government.

(3). The Problem of the Lack of Social Support

Although Guangzhou has formulated related policies about the training of the professional skills elevation for the migrant workers, only a minority really benefit from it. As most migrant workers are rejected outside the education welfare system, there exists the shortage of related social service and work aiming at the migrant workers' participation in the training of professional skill elevation; As the main provider of the vocational education, the openness of vocational colleges cannot meet the educational demand of the migrant workers. Due to the lack of service assurance system, the plan of vocational skill training for most migrant workers is suspended.

(4). The Problem of Inadequate Training System of Community Course

During the survey interview, we find that although some employment enterprise and subdistrict community can realize the meaning of carrying out the targeted community education training toward the migrant workers and also try to launch the relevant work by organizing various forces, but due to the lack of specialized organization which can take over the job professional and long-termly, the community course training cannot be designed concretely in coordination. It is hard for the community course with 'bad behavior' and 'one-hit wonders' to gain actual effect.

(5). The Lack of Learning Willingness of the Migrant Workers

In this investigation, we design the investigations of many projects including the current situation of the basic education reception, the requirement of spare-time culture and the main learning disabilities at present, etc. In particular, after a depth interview with the individual case of about 100 migrant workers, we know that the main obstacle of the migrant workers' inactive participation in the community course study comes from the following aspects: 'daily work at full capacity without time to study'; 'on the scale of study and making money, most respondents choose making money to reflect their utility in Guangzhou'. Guided by the idea of making money, they pursue the short-term interests and ignore badly the potential value of study; 'With poor cultural foundation, the migrant workers worry that they may fall behind with the study'. The migrant workers have fear toward the study because they lack the learning bases and ability. Most migrant workers still haven't still finished the senior high school or even junior high school, so there exist the lack of the bases for further learning objectively.

\section{The Suggestions based on the community Education Study and Development for the Migrant Workers.}

(1). Locate the community course education for the migrant workers correctly to make the community course accord with the requirement of life and employment. Elevating the employment and entrepreneurship is the important target location to satisfy the community study needs of the migrant workers.

Survey the learning demand of the migrant workers, research the current situation of profession and the future employment tendency and locate the education service according to the occupational demand. Stick to the orientation of professional demand and city life, design the vocational and technological education for the adults, develop the relevant courses of professional education, carry out the training project of practical skills and reform the traditional teaching mode. The community course training toward the migrant workers should 'accord with the professional characteristics, meet the employment needs, intensify the practical teaching and cultivate training skills'. Provide more choices and give more assistance to the migrant workers according to their learning needs to 
improve their basic cultural foundation and professional technology level. In this way, their employment and entrepreneurial ability can be enhanced comprehensively.

(2). Establishing the Specialized Agencies through Multi-lateral Cooperation and Strengthening the Overall Planning of the Community Education Course

As the investigation shows, the majority enterprise and community street have the willingness to launch the service and support for the community course, but the long-term existing shortage of the objective difficulties such as the leading of specialized departments, execution of specialized organization and the specialized personnel to take charge have inevitable influence on the planning and development of the community course. It's imperative to formulate the feasible policy measures, overall planning and increase the training intensity of the community public-good course; At the same time, reinforce the inspection intensity toward the community education training for the migrant workers launched by the employment units and advice the labor security department to take the lead and unite with related government departments such as industry and commerce, economic commission, education and street to establish a instruction center of professional skill for the migrant workers covering migrant workers from municipality, district and all places. Coordinate all the enterprises, industries, communities and various social training organizations to carry out the concrete business toward the professional training of the migrant workers, take full advantage of various media especially the information related to training and employment publicized by the new media and strengthen the inspection supervision of the training work and education quality.

(3). The Perfection of the Community Course Training Mechanism and the Establishment of a Perfect Expenditure and Evaluation System

1. Perfect the fund guarantee system. Suggest the implementation of the investment mechanism of community course training shared by the government, the employment units and the individual migrant worker based on investigation result.

2. Establish the assessment system. To motivate the enterprise's active support and investment in the community course, advice the related functional government department to make the participation rate, certification rate and employment rate of the migrant workers' community course training as the important assessment indicator and content of the enterprise assessing or annual inspection within the administration.

(4). Integrate the Resources of Vocational Training to Make Sure the Implementation of the Community Course Training for Migrant Workers.

Advice related departments to promote and support positively the higher vocational institutions and the training organizations with good qualification in the society to launch the professional skill training of the community course for the migrant workers and absorb the training contents, methods and efficiency of the higher vocational institutions and training organizations through the platform of the 'instruction center of the professional training for the migrant workers covering migrant workers from municipality, districts and all places' into the uniform standard management to assure the quality of education training.

2. Advice the higher vocational institutions and training organizations to shorten the time appropriately for the long-term training of the community course for the migrant workers, simplify the contents and carry out the work-study system for the migrant workers by adopting the method of 'class activities'.

(5). Increasing the Migrant Workers' Consciousness of Conscious Learning and Keeping Lifting the Employment Ability

This questionnaire investigation shows that there exist some situations that some migrant workers are not willing to study as they still have not realized the significance of study to the successful employment. It not only hinders their self-development, but also influences the sustained development of the economic society. The publicity education of the philosophy which is 'learning makes life happier' for the migrant workers should be strengthened and their consciousness of conscious learning should be improved. The whole society should use the incentives which can promote learning to motivate the migrant workers to 'learn for the city survival, learn for the improvement of life and learn for the career development'. In addition, the research about making 
the community course training for the migrant workers as the necessary condition to have a foothold in cities should be carried out.

\section{Acknowledgments}

This paper is 1 . The pilot project of the national education system reformation, 'pilot project of the learning society construction in Guangzhou', 'the subproject of the research', 'the research of investigation and development strategy based on the community education demand of the migrant workers in Guangzhou'(Project Number: sqxm2013019). 2. The scientific and sociological project in the municipal colleges in Guangzhou in 2013, 'advancement strategies research of the professional ability of the newly migrant works in Guangzhou’.(Project Number: 2012B010)

\section{References}

[1]. The Study of the Migrant Workers and Management Problems in Guangzhou. http://wenku.baidu.com/view/406a326548d7c1c708a14514.html [OL]. 2011-9-27

[2] Li Xungui, Liu Chujia, Zhao Xiaoduan, Pengqiang. The Status Investigation and Analysis of the Community Education Demand in Residential Community in Guangzhou[J]. Academic Research.2007.

[3] Zhao Hongya. The Historical Review of the Theoretical Research of American Adult Education in the $20^{\text {th }}$ Century[J] Journal of Hebei Normal University(Science and Education Press). 2007 